\title{
Eine Frage der Inkompetenz: Polizeiliche Warnungen vor Sexualstraftätern
}

Im Frühjahr 2009 beschäftigte ein Ereignis im Kreise Heinsberg über Tage hinweg die bundesdeutschen Medien. Stephan Pusch, der Landrat dieser Kommune, die für gewöhnlich wenig Anlass für überregionale Berichterstattung bietet, sprach mittels Presseerklärung eine öffentliche Warnung vor einem aus München frisch zugezogenen Neubürger aus. Dabei handelte es sich um einen soeben aus der Haft entlassenen Sexualstraftäter, der sich an Minderjährigen vergriffen hatte und als rückfallgefährdet eingestuft wurde. ${ }^{1}$ Ein kurzer Text, der grundlegende Rechtsfragen aufwirft.

Staatliches Informationshandeln wird in der wissenschaftlichen Literatur häufig positiv konnotiert: Viele sehen darin eine der sichtbarsten Ausprägungen des Gewährleistungsstaats, der sich der beschränkten Wirksamkeit hoheitlicher Steuerung durch „command and control“ bewusst ist und stattdessen auf die Einsicht seiner Bürger und ihre Fähigkeit zur Selbstorganisation setzt. ${ }^{2}$ Nach $U d o$ Di Fabio bringt dieser mal empfehlende, mal warnende „Präzeptorstaat“ den aufklärerischen Gedanken des selbstbestimmten Individuums erst richtig zur Geltung, indem er den mündigen Bürger in die Lage versetzt, eigenverantwortlich zu handeln. ${ }^{3}$ Dieses Bild von einer Verwaltung, die postmoderne Ungewissheit durch intelligente Handlungsformen in den Griff bekommen mag, wird nun durch die Warnung des Landrats von Heinsberg auf die Probe gestellt. War sie ein notwendiger Akt der Fürsorge, um die Bevölkerung zu beruhigen und an ihre Eigenvernunft zu appellieren ${ }^{4}$ Oder die übereilte Maßnahme eines Lokalpolitikers, die seiner Popularität mehr zugute kam als der Sicherheit? Mancher mag sich an das längst vergessen geglaubte Institut des Prangers erinnert gefühlt haben, durch das in der Frühneuzeit Ehrenstrafen vollstreckt und der soziale $\mathrm{Zu}$ sammenhalt im Wege negativer Abgrenzung gestärkt wurde. ${ }^{5}$

Jenseits solch zugegeben grobschlächtiger Klassifikationen verdient der Fall gesteigerte juristische Aufmerksamkeit. Denn die Forderung nach einem besseren Schutz vor Sexualstraftätern und insbesondere solchen mit pädophiler Neigung ist verbreitet. Die Einführung einer nachträglichen Sicherungsverwahrung durch den Bundesgesetzgeber im Jahr 2004 und selbst die mehrfache Schließung vermeintlicher „Lücken“ im Regelwerk, ${ }^{6}$ zuletzt durch Einführung einer nachträg-

* Für Kritik und Anregungen danke ich Dorothea Barck, Jürgen Bast, Jochen von Bernstorff, Felix Hanschmann, Diana Zacharias, sowie der Redaktion.

1 Pressemitteilung der Polizeibehörde des Landkreises Heinsberg, 2.3.2009, http://www.polizei-nrw.de/ presseportal/behoerden/heinsberg/article/meldung-090302-152754-74-499.html.

2 C. Bumke, Publikumsinformation. Erscheinungsformen, Funktionen und verfassungsrechtlicher Rahmen einer Handlungsform des Gewährleistungsstaates, Die Verwaltung 37 (2004), S. 3.

3 U. Di Fabio, Grundrechte im präzeptoralen Staat am Beispiel hoheitlicher Informationstätigkeit, JZ 1993, S. 689 (691).

4 So scheinbar die offizielle Begründung, vgl. „Die Hysterie kam trotzdem“, FAZ vom 9.3.2009.

5 R. van Dülmen, Entstehung des frühneuzeitlichen Europa, Band 24, 1550-1648, 9. Aufl. 2000, S. 239.

$6 \$ 66$ b StGB, vgl. Gesetz zur Einführung der nachträglichen Sicherungsverwahrung vom 23.7.2004, BGBl. I, S. 1838, zuletzt geändert durch das Gesetz zur Reform der Führungsaufsicht und zur Änderung der Vorschriften über die nachträgliche Sicherungsverwahrung vom 13.4.2007, BGBl. I, S. 513. Eine Übersicht über die kontinuierliche Ausweitung der Sicherungsverwahrung durch den Gesetzgeber findet sich bei J. Kinzig, Schriftliche Stellungnahme für die öffentliche Anhörung des Rechtsausschusses 

nicht beruhigen. ${ }^{8}$ Man erinnere sich nur an den öffentlichen Protest im Jahr 2007 anlässlich der Haftentlassung eines Sexualverbrechers, der in Quedlinburg Wohnung nahm. ${ }^{9}$ Die Öffentlichkeit will sich vor diesen „tickenden Zeitbomben“ aber auch selbst schützen können. Dazu verlangt sie nach staatlichen Warnungen. ${ }^{10}$ Zunehmend findet sie damit bei Entscheidungsträgern Gehör. ${ }^{11}$ Andere Staaten sind uns dabei weit voraus. In den Vereinigten Staaten beschlossen zahlreiche Staaten sog. „Megan’s Laws“, Gesetze, die neben einer Meldepflicht für freigelassene Sexualstraftäter oft auch die öffentliche Bereitstellung dieser Informationen vorsehen. ${ }^{12}$ Heute kann sich der besorgte Bürger dort in vielen Landesteilen per Internet darüber informieren, wo und vor wem er oder seine Kinder sich besser in Acht nehmen sollten - es bleiben oft nicht viele "go areas“ übrig. ${ }^{13} \mathrm{Ob}$ dadurch auch nur ein einziger Missbrauch verhindert wurde, entzieht sich begreiflicherweise der statistischen Erfassung. Dokumentiert hingegen sind Fälle von Lynchjustiz gegen einige der in den Verzeichnissen geführten entlassenen Täter. ${ }^{14}$ Selbst ohne öffentliches Verzeichnis sind solche Täter ja bereits mitunter der Selbstjustiz ihrer Mitbürger ausgeliefert. ${ }^{15}$

Es drängt sich daher die juristische Fragestellung auf, ob und was die Bevölkerung über die Vergangenheit ihrer Mitbürger in Erfahrung bringen können soll. Wissen ist Macht - ein staatliches Wissensmonopol entmündigt den Bürger und erschwert seinen eigenverantwortlichen Umgang mit Gefahren. Auf der anderen Seite ist nicht alles Wissen immer legitim, insbesondere solches, das die Ansehung unserer Mitmenschen betrifft. Nicht von ungefähr trägt Justitia eine Augenbinde. Dieses Spannungsverhältnis bildet den Hintergrund der folgenden Erörterungen. Ziel dieses Beitrags ist es hingegen nicht, ein Urteil über die Rechtmäßigkeit der Presseerklärung von Heinsberg zu fällen. Allerdings informiert dieser Vorgang über die faktischen und rechtlichen Schwierigkeiten, die bei der Erörterung des rechtlichen Rahmens polizeilicher Warnungen vor entlassenen Sexualstraftätern zu berücksichtigen sind. Daher folgt zunächst ein Überblick über den Fall Heinsberg (I.), dem sich eine Erörterung des rechtlichen Rahmens polizeilicher War-

des Deutschen Bundestages am 28.5.2008, http://www.bundestag.de/ausschuesse/a06/anhoerungen/ Archiv/37_Jugendstrafrecht-Sichverw/04_Stellungnahmen/Stellungnahme_Kinzig.pdf, S. 4-5.

7 Gesetz zur Einführung der nachträglichen Sicherungsverwahrung bei Verurteilungen nach Jugendstrafrecht vom 8.7.2008, BGBl. I, S. 1212.

8 Neue „Lücken“ im Recht der Sicherungsverwahrung sind bereits entdeckt worden, vgl. „Nachbarn sollen vor Sexualstraftäter gewarnt werden“, FAZ vom 26.10.2008.

9 Weitgehend unbemerkt blieb, dass die Polizei in diesem Fall inzwischen die Führungsaufsicht über den Täter beenden konnte. Es hatte sich gezeigt, dass von ihm keine akute Gefahr ausging. Vgl. „Auf Schritt und Tritt überwacht", Süddeutsche Zeitung vom 3.3.2009.

10 Vgl. „Nachbarn sollen vor Sexualstraftäter gewarnt werden“, FAZ vom 26.10.2008.

11 Im Zuge des Aufbaus einer zentralen Datei über haftentlassene, rückfallgefährdete Sexualstraftäter in Bayern wurde diskutiert, ob diese Daten nicht auch zur Information und Warnung der Öffentlichkeit genutzt werden können, vgl. 22. Tätigkeitsbericht des Landesdatenschutzbeauftragten, Bayerischer Landtag, Drucksache 15/6700 vom 29.1.2007, S. 15. Die Datei ist auf das Polizeiaufgabengesetz gestützt.

12 Überblick zur Gesetzeslage in den US-Bundesstaaten auf http://www.meganslaw.org.

13 Vgl. z.B. die entsprechende Website des Staats Pennsylvania mit Fotos und persönlichen Angaben zu verurteilten Sexualstraftätern sowie lediglich rudimentären Informationen zu ihren Delikten, die teilweise weit über 20 Jahre zurück liegen, http://www.pameganslaw.state.pa.us. Nicht nur schwere Verbrechen, auch bereits der bloße Besitz von Kinderpornographie führen zu einem Eintrag in dem Register. Je nach Art oder Anzahl der Verbrechen wird der Eintrag u.U. gar nicht mehr gelöscht. Angaben zur Einschätzung der aktuellen Gefährlichkeit fehlen hingegen.

14 Einen bedrückenden Fall schildert der Artikel „Sex Offender Registries: Putting Lives at Risk?“, 18.4.2006, http://abcnews.go.com/US/story?id=1855771\&page=1.

15 Die Hybris geht so weit, dass kürzlich in einer deutschen Haftanstalt ein verurteilter Mörder sich moralisch dazu berufen fühlte zu versuchen, einen ebenfalls verurteilten Kindermörder zu töten. Das Gesicht des Kindermörders wurde dabei schwer entstellt. 
nungen vor Sexualstraftätern anschließt (II.). Der Schwerpunkt liegt hierbei auf den Fragen der gesetzgeberischen Kompetenz und der Verhältnismäßigkeit.

\section{Hintergrund: Der Fall Heinsberg}

Anfang März zog der soeben aus der Justizvollzugsanstalt Straubing entlassene Karl D. in den Ortsteil Randerath der Gemeinde Stadt Heinsberg, Kreis Heinsberg, Nordrhein-Westfalen, wo er im Haus der Familie seines Bruders aufgenommen wurde. Karl D. wurde bereits in den Achtzigern wegen der Vergewaltigung einer 17-jährigen zu 5 Jahren Freiheitsstrafe verurteilt, wovon er 3 Jahre und 8 Monate absaß. Zuletzt saß er wegen der Vergewaltigung zweier 14 und 15 Jahre alter Mädchen. Diese Taten zeichneten sich durch ein hohes Maß an Grausamkeit aus, u.a. traktierte der Täter die Geschlechtsteile der Opfer mit Nadeln und setzte sie nachts gefesselt aus. Dafür hatte Karl D. 14 Jahre Freiheitsstrafe bekommen und voll verbüßt. In dem Urteil wurde keine anschließende Sicherungsverwahrung angeordnet. Karl D. stritt die Taten ab und weigerte sich während der Haft, sich einer Therapie zu unterziehen. Als die Haftentlassung anstand, beantragte die Staatsanwaltschaft München nachträgliche Sicherungsverwahrung nach $\$ 66$ b StGB. Trotz zweier Gutachten, in denen Karl D. eine hohe Rückfallgefährdung sowie eine Neigung zu Sadismus attestiert wurden, gab das Landgericht München dem Antrag der Staatsanwaltschaft nicht statt. \66 b Abs. 1 StGB verlangt, dass zusätzlich zu der negativen Gefahrprognose nach der Verurteilung Tatsachen erkennbar geworden sind, die auf eine erhebliche Gefährlichkeit des Verurteilten für die Allgemeinheit hinweisen (sog. „Nova“). Derartige Tatsachen konnte das Gericht nicht feststellen, was später vom Oberlandesgericht in zweiter Instanz bestätigt wurde. Daher ordnete es die Haftentlassung sowie die in einem solchen Fall obligatorische Führungsaufsicht an und erließ entsprechende Weisungen. ${ }^{16}$ Inzwischen war der Fall publik geworden. Die Boulevardpresse schien sogar den neuen Aufenthaltsort von Karl D. ausfindig gemacht zu haben. Deswegen sollen sich am 1. März bereits Kamerateams in Heinsberg aufgehalten haben. Am Montag, dem 2. März 2009, veröffentlichte Landrat Pusch als Leiter der Kreispolizeibehörde eine kurze Pressemitteilung, um „die Bevölkerung im Kreis Heinsberg sachlich über einen Vorgang zu informieren, mit dem wir seit dem Wochenende im Kreis Heinsberg konfrontiert sind. "17 Darin werden der Vorname sowie der abgekürzte Nachname des Täters und der betroffene Ortsteil genannt. Ferner schildert der Landrat die Taten von Karl D. in manchem Detail. So heißt es z.B.: „Aufsehen erregten die Vergewaltigungsfälle des heute 57-jährigen durch das hohe Maß an Brutalität und Grausamkeit, mit der die Opfer misshandelt wurden. "Sodann drückt der Landrat sein „Unbehagen“ über die Ablehnung der nachträglichen Sicherungsverwahrung aus und schließt sich der Kritik des bayerischen Innenministers an dieser Entscheidung an. Schließlich versichert der Landrat, alle notwendigen Maßnahmen zu treffen, um die Sicherheit der Bevölkerung zu gewährleisten. Einzelheiten nennt er aus polizeitaktischen Gründen nicht. Eine Aufforderung, wie sich die Bevölkerung verhalten möge, etwa eine Ermahnung zu Ruhe und Besonnenheit, sucht man in der Mitteilung vergeblich.

Zwar mag die Presseerklärung nicht allein ursächlich gewesen sein, dass der Aufenthaltsort von Karl D. weithin bekannt wurde. Medial zündete sie aber eine 
Bombe. Nahezu jeder der zahlreichen Presseberichte zu diesem Fall, die in den folgenden Wochen erschienen, nimmt Bezug auf diese Erklärung des Landrats. Umgehend begannen Demonstrationen vor dem schnell identifizierten Haus des Bruders von Karl D. Daran nahmen auch viele Ortsfremde teil. Größtenteils handelte es sich um unorganisierte Aktionen betroffener Bürger. Fotos von Karl D. mit einem schwarzen Balken vor den Augen wurden überall im Ort ausgehängt. Auch die NPD nutzte die Situation, um mit einer Demonstration in der Ortsmitte ihrer Forderung nach der Todesstrafe für Kinderschänder Gehör zu verschaffen. Als die NPD-Anhänger entgegen der Demonstrationsanmeldung durch den Ort zum Haus des Bruders von Karl D. vordringen wollten, griff die Polizei ein und nahm 63 Personen fest. ${ }^{18}$ Angesichts dieser Vorgänge forderte der Ortsvorsteher von Randerath in einem Schreiben seine Mitbürger zur Ruhe und Beendigung der Aktionen auf. Der Täter traute sich nicht mehr aus dem Haus, sein Bruder wurde im April vorübergehend in eine psychiatrische Anstalt eingewiesen, nachdem er gegenüber einem Polizeibeamten ausfällig geworden war. ${ }^{19}$

Auf Anfrage des Landesdatenschutzbeauftragten überprüfte das nordrheinwestfälische Innenministerium den Fall. Es kam zu dem Schluss, die Presseerklärung sei rechtmäßig gewesen, da die Öffentlichkeit bereits Wind von den darin verlautbarten Fakten bekommen hätte. Sollte dies tatsächlich so gewesen sein, sollte der Landrat nur das verbreitet haben, was ohnehin schon bekannt war, so verwundert es zumindest, dass nun gerade Landrat Pusch, der im September 2009 zur Wiederwahl antritt, von der Bevölkerung viel Lob für sein mutiges und entschiedenes Handeln zugesprochen bekommt. Vielleicht verdient es eher die Boulevardpresse für ihre mutige Berichterstattung. - Doch allein der unerschrockene Mut zur Wahrheit kann Unrecht nicht zu Recht machen. Daher scheint ein genauer Blick auf den rechtlichen Rahmen von staatlichen Warnungen über zuziehende entlassene Sexualstraftäter angebracht.

\section{Rechtlicher Rabmen polizeilicher Warnungen vor Sexualstraftätern}

So groß die Unterschiede zwischen den verschiedenen Arten staatlicher Informationsakte auch sein mögen, seien es Warnungen, Empfehlungen ${ }^{20}$ oder auch nur statistische Daten, ${ }^{21}$ ihnen allen ist gemeinsam, dass sie nur indirekt wirken und unmittelbar kein bestimmtes Verhalten vorschreiben. Dennoch können sie durchaus erhebliche, wenngleich nicht präzise steuerbare Wirkungen entfalten. ${ }^{22}$ Dieser Umstand stellt die im Grundsatz auf rechtsförmige Akte ausgerichtete Dogmatik des öffentlichen Rechts regelmäßig vor besondere Herausforderungen, wenngleich sie inzwischen reichlich Erfahrung mit Informationsakten gesammelt hat. Danach lassen sich grob drei Kategorien bilden: ${ }^{23} \mathrm{Zum}$ einen können öffentliche Stellen die Öffentlichkeit über ihre Tätigkeit informieren und so eine wichtige Voraussetzung für die demokratisch notwendige Mei-

18 Pressemitteilung der Kreispolizeibehörde Heinsberg vom 7.3.2009, http://www.polizei-nrw.de/presseportal/behoerden/heinsberg/article/meldung-090307-195944-84-506.html.

19 Aachener Zeitung vom 17.4.2009, http://www.az-web.de/lokales/euregio-detail-az/867016.

20 Dazu Bumke (Fn. 2).

21 Im Kontext des Völkerrechts A. von Bogdandy/M. Goldmann, Die Ausübung internationaler öffentlicher Gewalt durch Politikbewertung. Die PISA-Studie der OECD als Muster einer neuen völkerrechtlichen Handlungsform, ZaöRV 69 (2009), S. 51-102.

22 C. Gusy, Die Informationsbeziehungen zwischen Staat und Bürger, in: W. Hoffmann-Riem/E. Schmidt-Aßmann/A. Voßkuhle (Hrsg.), Grundlagen des Verwaltungsrechts, Bd. 2, 2008, S. 221-304, Rn. 101.

23 Gusy (Fn. 22), Rn. 98, 102, 104. 
nungsbildung schaffen. Diese Informationstätigkeit ist von ihren Kompetenzen implizit miterfasst. Zweitens können sie Empfehlungen aussprechen, um eine Verhaltenssteuerung durch positive Anreize zu bewirken. Wenngleich solche Empfehlungen sich etwa bei Produkten negativ auf Konkurrenten auswirken mag, wird darin kein Grundrechtseingriff gesehen. Sofern die Empfehlung sachlich gerechtfertigt ist, werden die Auswirkungen auf Konkurrenten als zu indirekt erachtet. Ist ein Individuum hingegen wie Karl D. direkt und negativ von einer staatlichen Warnung betroffen, stellt sich die Frage eines Grundrechtseingriffs (1.) sowie der Anforderungen an seine Rechtfertigung (2. und 3.).

\section{Warnung als Eingriff in das informationelle Selbstbestimmungsrecht}

Die Rechtsprechung hat sich bereits mehrfach mit der grundrechtlichen Wirkung staatlicher Warnungen auseinandergesetzt und zeigt nunmehr große Zurückhaltung bei der Annahme eines Eingriffs in Grundrechte. In der TransparenzlistenEntscheidung wurde ein Eingriff in die Berufsfreiheit noch angenommen, wenn die staatliche Informationstätigkeit auf eine entsprechende Veränderung der Marktbedingungen für die betroffenen Produkte abzielte. ${ }^{24}$ In der Glykol-Entscheidung lehnte das Bundesverfassungsgericht hingegen eine Beeinträchtigung der Berufsfreiheit durch Produktwarnungen rundweg ab, sofern eine öffentliche Stelle im Rahmen ihrer sachlichen Zuständigkeit objektiv informiert. ${ }^{25}$ Dahinter steht der Gedanke, dass der Ruf eines Produkts erst in einem kommunikativen Prozess konstituiert wird. Staatliche Informationen sind für diesen Prozess notwendig, da der Markt allein nicht immer für ausreichende Informationen sorgt. Was aber erst konstitutiv für den Ruf ist, kann ihn nicht zugleich beeinträchtigen. ${ }^{26}$ In der Osho-Entscheidung konzedierte dasselbe Gericht dann, dass eine Warnung vor einer religiösen Gemeinschaft zu einer „mittelbar-faktischen Grundrechtsbeeinträchtigung “ führe. Allerdings sei der Gesetzesvorbehalt nicht im selben Umfang wie der Schutzbereich der Grundrechte gewachsen. Daher genüge es für die Verfassungsmäßigkeit religionsbezogener Äußerungen, dass die staatliche Zuständigkeitsordnung eingehalten und das Neutralitätsgebot nicht verletzt werde. ${ }^{27}$ Die Rechtsprechung dosiert somit auch den Gesetzesvorbehalt in diesem Kontext recht sparsam.

Die Verbreitung von Informationen über entlassene Sexualstraftäter dürfte demgegenüber ein neues Kapitel in der Beurteilung staatlicher Warnungen aufschlagen. Es geht nun nicht mehr um die Warnung vor einem bestimmten Gegenstand oder der Tätigkeit einer Gruppe. Der Grund der Warnung liegt vielmehr in der Person selbst, und die Warnung beinhaltet somit notwendigerweise die Veröffentlichung personenbezogener Daten. Damit wird sie zu einem Fall für das informationelle Selbstbestimmungsrecht. Im Unterschied $\mathrm{zu}$ der in der soeben aufgeführten Rechtsprechung thematisierten Berufs- oder Religionsfreiheit geht das informationelle Selbstbestimmungsrecht über einen bloßen Schutz vor Exzessen durch Fehlinformationen oder Diffamierungen, wie sie etwa der strafrechtliche Ehrschutz schon seit langem gewährt, hinaus und ermöglicht dem Einzelnen, die Erhebung, Verarbeitung und Veröffentlichung personenbezogener Daten recht weitgehend zu kontrollieren. Nur wer diese Kontrolle hat, kann einigermaßen unbeschwert seine sozialen Beziehungen organisieren. ${ }^{28}$ 
Die Veröffentlichung des Aufenthaltsortes eines aus der Haft entlassenen Sexualstraftäters greift in den Schutzbereich des informationellen Selbstbestimmungsrechts ein, und zwar direkt und unmittelbar, obgleich es sich um einen Informationsakt handelt. Denn das informationelle Selbstbestimmungsrecht ist auf Informationsakte geradezu gemünzt, es lässt sich daher klar zwischen Schutzbereich, Eingriff und Rechtfertigung differenzieren..$^{29} \mathrm{Ob}$ die veröffentlichten Informationen bereits dritten Personen, etwa der Presse, bekannt sind, dürfte für die Frage des Eingriffs unerheblich sein. Denn zum einen gibt die staatliche Bestätigung eines bereits bekannten oder vermuteten Faktums diesem eine neue Qualität. Zum andern dürfte aber selbst in dem Moment, wo persönliche Daten bereits weite Verbreitung gefunden haben und die betroffene Person zum Gegenstand einer öffentlichen Debatte geworden ist, noch ein unmittelbarer Eingriff vorliegen. Eine schlichte Übertragung der Glykol- und Osho-Rechtsprechung, die dem Staat zu Recht eine gewichtige Rolle in öffentlichen Debatten zuweist, scheidet aus. Denn in diesen Situationen waren die in Frage stehenden Grundrechte durch die Informationsakte von vornherein nicht beeinträchtigt. Diese Grundrechte schützen eben nicht dezidiert vor der Verbreitung von Informationen, wie das informationelle Selbstbestimmungsrecht. Und der Schutzbereich des Letzteren erstreckt sich nicht etwa auf „geheime“ Informationen, sondern auf personenbezogene Informationen. Würden nur nicht-öffentliche Informationen geschützt, bedeutete dies eine Kapitulation vor der normativen Kraft des Faktischen sowie eine Einladung zur Umgehung durch Indiskretionen. Allerdings ist im Rahmen der Rechtfertigung zwischen öffentlichen und nichtöffentlichen Informationen zu differenzieren.

\section{Gesetzesvorbehalt und Gesetzgebungskompetenz}

Beeinträchtigungen der informationellen Selbstbestimmung müssen nach dem Bundesverfassungsgericht stets eine Grundlage in einem förmlichen Gesetz finden, an dessen Bestimmtheit hohe Anforderungen gestellt werden. ${ }^{30}$ Ein Rückgriff auf die polizeiliche Generalklausel scheidet daher aus. Die Landespolizeigesetze enthalten aber spezifische Befugnisse, die die Datenübermittlung an Personen oder an Stellen außerhalb des öffentlichen Bereichs regeln, wie etwa $\$ 29$ Abs. 1 des Polizeigesetzes Nordrhein-Westfalen (NWPolG). Der Gesetzgeber mag bei Erlass dieser Normen zwar anders gelagerte Situationen im Blick gehabt haben; eine Anwendung des $\$ 29$ Abs. 1 NWPolG auf öffentliche Warnungen vor Sexualstraftätern erscheint mit dem Wortlaut der Vorschrift aber nicht grundsätzlich unvereinbar. Dann käme es darauf an, ob bereits allein im Zuzug eines als rückfallgefährdet eingestuften Sexualstraftäters eine konkrete Gefahr liegt.

Doch die Anwendung präventiv-polizeilicher Befugnisse scheitert bereits daran, dass den Ländern überhaupt keine Kompetenz zur gesetzlichen Regelung präventiver Maßnahmen gegen einen aus der Haft entlassenen, unter Führungsaufsicht stehenden Straftäter zusteht. Zwar sind sie grundsätzlich zur Regelung der Gefahrenabwehr befugt. Allerdings verleiht Art. 74 Abs. 1 Nr. 1 GG dem Bund die konkurrierende Gesetzgebungskompetenz für das Strafrecht. Dies umfasst auch die in $\mathbb{\$} 61 \mathrm{ff}$. StGB geregelten Maßregeln der Besserung und Sicherung. Die darin normierten Befugnisse, unter denen sich keine Ermächtigung zur Inmittelbar“ im Rahmen der Beurteilung staatlicher Informationsakte, dazu kritisch D. Murswiek, Staatliche Warnungen, Wertungen, Kritik als Grundrechtseingriffe, DVBl. 1997, S. 1021 (1022).

So das Volkszählungsurteil, BVerfGE 65, 1 . 
formation nicht-öffentlicher Stellen findet, enthalten eine abschließende Regelung der Maßnahmen, die nach Haftentlassung zum Zweck des Schutzes der Allgemeinheit vor künftigen Straftaten des Entlassenen ergriffen werden dürfen. Der abschließende Charakter der $\$ \int 61 \mathrm{ff}$. StGB wurde vom Bundesverfassungsgericht bereits 2004 in Bezug auf die - damals noch nicht im StGB geregelte nachträgliche Sicherungsverwahrung festgestellt. Unter den Begriff des Strafrechts in Art. 74 Abs. 1 Nr. 1 GG fielen alle, auch nachträgliche, Maßnahmen repressiver oder präventiver Art, „die an die Straftat anknüpfen, ausschließlich für den Straftäter gelten und ihre sachliche Rechtfertigung auch aus der Anlasstat beziehen." Dies umfasse auch die Regelung von Unrechtsfolgen, die nicht die Schuld des Täters vergelten, somit sämtliche Maßregeln der Besserung und Sicherung. ${ }^{31}$ Zur Begründung stützt sich das Gericht vor allem auf die historische Interpretation des Begriffs.

Diese Rechtsprechung lässt sich aus mindestens zwei Gründen auf die hier interessierende Konstellation öffentlicher Warnungen vor Sexualstraftätern übertragen. Zum einen hat der Bundesgesetzgeber die Bestimmungen über Maßregeln der Besserung und Sicherung seit der Entscheidung des Bundesverfassungsgerichts im Jahr 2004 mehrfach neu geregelt. Ihm war dabei bekannt, dass das Bundesverfassungsgericht ihm die konkurrierende Gesetzgebungskompetenz für sämtliche Maßnahmen zuweist, die an die Straftat anknüpfen. Eine Rechtsgrundlage für die Information der Öffentlichkeit wurde dabei nicht geschaffen. Vor dem Hintergrund, dass die Forderung nach öffentlichen Warnungen nicht erst seit dem Fall Heinsberg immer wieder erhoben worden ist, kann dies nur dahingehend bewertet werden, dass eine bewusste Lücke gelassen wurde, weil eine Information der Öffentlichkeit nicht gewollt war. Dem lässt sich ein pragmatisches Argument hinzufügen. Die Anordnung von Maßregeln der Besserung und Sicherung obliegt dem Gericht des ersten Rechtszuges, also derjenigen staatlichen Stelle, die mit dem betroffenen Straftäter und seinen Taten womöglich noch vertraut ist und sich vor allem dank des Verfahrens gemäß $\$ 275$ a StPO ein gutes Bild über seine Resozialisierungschancen machen kann, zugleich aber über die notwendige Distanz verfügt, um eine unabhängige Entscheidung zu treffen, die auch die Sicherheitsinteressen der Bevölkerung berücksichtigt. Weder ein kommunaler Amtsträger noch die Polizei noch der Gefängnisdirektor oder der psychologische Gutachter sind auf einem vergleichbaren Wissensstand bzw. können die widerstreitenden Interessen derart umfassend in den Blick nehmen.

Somit schließen die $\$ \mathbb{S} 61 \mathrm{ff}$. StGB die Anwendung der Landespolizeigesetze für öffentliche Warnungen vor entlassenen Sexualstraftätern aus, sofern die Warnungen nur an die Straftaten oder an Ereignisse vor oder während der Strafvollstreckung anknüpfen und unabhängig von Ereignissen nach der Haftentlassung ergehen sollen. Dem Landesgesetzgeber fehlt es an der Gesetzgebungskompetenz hinsichtlich aller präventiven Maßnahmen, die nur an verbüßte Vorstrafen anknüpfen.

Dies bedeutet nicht, dass die Polizei tatenlos zusehen müsste, wie ein entlassener Sexualstraftäter sich zur Begehung neuer Straftaten anschickt. Denn zum einen gibt es ja die Führungsaufsicht, und die Einhaltung der in diesem Rahmen erteilten Weisungen darf überwacht und durchgesetzt werden. Zum anderen darf die Polizei selbstverständlich aufgrund Landesrechts tätig werden, sobald eine neue Situation eintritt. Um eine völkerrechtliche Metapher zu bemühen: während allein auf das Wissen um die Rückfallgefahr gestützte präemptive Eingriffe der Sicherheitsbehörden nicht aufgrund des Landesrechts möglich sind, können 
die Sicherheitsbehörden sehr wohl aufgrund des Polizeirechts präventiv tätig werden, sofern neue Tatsachen eine konkrete Gefahr begründen. Wenn sich also ein entlassener Sexualstraftäter z.B. an Schulen oder Kindergärten in verdächtiger Weise herumtreibt, darf die Polizei aufgrund landesrechtlicher Befugnisse einschreiten. Denn dann wird nicht ausschließlich an das durch frühere Straftaten erworbene Sonderwissen angeknüpft. Eingriffe kommen auch in Frage, wenn etwa die Unruhe in der Bevölkerung die Schwelle der konkreten Gefahr überschreitet und die Behörden zu ihrer Beruhigung eine Mitteilung herausgeben, die personenbezogene Daten enthält, um etwa Überreaktionen wie Übergriffe auf den Entlassenen oder Ausschreitungen bei Protestkundgebungen zu verhindern. Dabei sind aber zwei Einschränkungen zu beachten. Zum einen darf die Behörde die öffentliche Diskussion nicht selbst entfachen oder öffentliche Unruhe zu großzügig antizipieren, etwa durch frühzeitige Presseerklärungen, die vorrangig auf Information, nicht auf Diskussion angelegt sind. Die Kompetenzgrenze gebietet jedem Aktionismus Einhalt. Zum andern ist der Betroffene in dieser Konstellation gemäß der Theorie der unmittelbaren Verursachung 32 als Nichtstörer zu behandeln, sofern er nicht durch sein Verhalten nach der Entlassung zur Entstehung der Unruhe beigetragen hat. Denn die Gefahr entsteht durch das zurechenbare Dazwischentreten Dritter. Damit gelten die in den Polizeigesetzen konkretisierten besonderen Voraussetzungen an die Erforderlichkeit und Angemessenheit der Inanspruchnahme eines Nichtstörers.

\section{Verhältnismäßigkeit polizeilicher Warnungen}

Sollten im Rahmen der soeben beschriebenen, eng umrissenen präventiv-polizeilichen Befugnisse polizeiliche Warnungen erwogen werden, so ist dabei der verfassungsmäßige Grundsatz der Verhältnismäßigkeit zu beachten. Das Sicherheitsinteresse fließt dabei als ein Aspekt unter mehreren in die Abwägung ein. Daher sollte man sich zu allererst fragen, welchen Sicherheitsgewinn denn eine öffentliche Warnung vor einem zuziehenden Sexualstraftäter bringt. Welches Verhalten soll die Bevölkerung aufgrund der Warnung an den Tag legen? Wie soll sie etwa ihre Kinder schützen? Indem sie permanent in Angst lebt und sie dauerhaft nicht mehr ohne Aufsicht lässt? Das kann keine langfristige Lösung sein. Indem sie sich bewaffnet? Gott bewahre. Im Fall Heinsberg wurde zudem nur das Initial des Nachnamens des Betroffenen angegeben, kein Foto beigefügt, der Wohnort nur mit dem Stadtteil angegeben. Dies mag dem Betroffenen zwar einen letzten Rest an Persönlichkeitsschutz gewähren, allerdings kann mit diesen Informationen niemand sich effektiv schützen, außer Eingeweihten, die wissen, um wen es sich handelt. Besteht nicht Grund zu der Annahme, dass dies bei denen, die den Täter nicht genau kennen, ein ungeheures Misstrauen schürt, dass sie in jedem ihnen unbekannten Mann entsprechenden Alters den Täter vermuten? Dass dann versteckte Verdächtigungen und Gerüchte die öffentliche Atmosphäre prägen? Außerdem: Wer sagt denn, dass der Betroffene nur an seinem Wohnort seine nächste Tat begehen wird? Er ist frei und könnte zum Zwecke seines nächsten Verbrechens auch in die näher oder weiter entfernte Umgebung fahren. Wie schützt man die dortigen Bürger? Müsste dann nicht eine bundesoder europaweite Warnung her unter Offenlegung aller Daten und mit Bild? Aber wäre eine solche Maßnahme angemessen angesichts der nur vagen Aussicht, damit ein weiteres Verbrechen zu verhindern? 
Spätestens an dieser Stelle sollte der Einsatz milderer Mittel erwogen werden. Hierbei ist nicht nur das Potenzial der zahlreichen, im Rahmen der Führungsaufsicht ( $\$ \int 68 \mathrm{ff}$. StGB) möglichen Maßnahmen zu berücksichtigen, ${ }^{33}$ sondern auch ergänzende präventive Mittel, sofern neue Tatsachen ihre Anwendung rechtfertigen. Die Polizeibehörden haben gute Erfahrung mit der sog. Gefährderansprache gemacht. Dies stellt eine wenig grundrechtsinvasive Maßnahme dar, ${ }^{34}$ durch die der Täter mit seiner Situation konfrontiert wird und signalisiert bekommt, dass er seitens der Behörden, und zwar auch derer, die nicht die Maßregeln überwachen, als Problem wahrgenommen wird, dass er gewissermaßen „auf der Liste“ steht. Darüber hinaus kommen Maßnahmen wie Platzverweise oder Ingewahrsamnahme in Betracht. Sollte auch dies nicht ausreichend erscheinen, so muss noch immer nicht gleich öffentlich gewarnt werden. Stattdessen könnten z.B. die Leiter umliegender Schulen oder Kindergärten vertraulich informiert werden.

Die grundrechtliche Abwägung hat ferner das Resozialisierungsinteresse des Straftäters zu berücksichtigen. Der Täter hat ja seine Strafe abgesessen, die Gesellschaft sollte ihm also einen neuen Start ermöglichen. Gerade bei Sexualstraftätern und insbesondere solchen, die sich an Kindern vergriffen haben, stellt aber die große gesellschaftliche Ächtung, die ihnen entgegengebracht wird, bereits ein großes Resozialisierungshindernis dar. Durch Warnungen vor dem Täter (insbesondere solche, die mit keinem Wort zu Ruhe und Besonnenheit ermahnen) wird dieser Effekt nur verstärkt. Eine Bekanntgabe von Namen und Wohnort kann ganz verheerende Konsequenzen nicht nur für die Wiedereingliederung des Täters in die Gesellschaft, sondern bereits für seine schiere Sicherheit haben. Das zeigte nichts deutlicher als der NPD-Marsch zum Haus des Karl D. in Heinsberg. Die Polizei schien zeitweilig mehr damit beschäftigt, den Täter vor der Öffentlichkeit zu schützen, als umgekehrt. Man könnte daher sagen, dass gerade die Täter von gesellschaftlich stark geächteten Taten nach Verbüßung ihrer Strafe einen besonders hohen Schutz vor Veröffentlichung ihrer Daten zugestanden bekommen sollten. Sie sind wahrscheinlich größeren Repressalien ausgesetzt als andere entlassene Straftäter. Die Irrationalität des Sicherheitsgefühls der Bevölkerung verlangt nach einer Erhöhung der Eingriffsschwelle, nicht nach einer Absenkung. ${ }^{35}$ Dazu kommt, dass sich Informationen nicht einfach zurückholen lassen, wie sich etwa ein Verwaltungsakt widerrufen lässt. Stellt sich z.B. heraus, dass von dem entlassenen Straftäter nur noch eine geringe Gefahr ausgeht, geringer als ursprünglich angenommen, oder stellt sich gar die ursprüngliche Prognose als falsch heraus, lässt sich die Warnung nicht mehr ungeschehen machen; sie wird den Betroffenen Zeit seines Lebens verfolgen. Wahrscheinlich finden die hohen Eingriffsvoraussetzungen des Rechts auf informationelle Selbstbestimmung genau in dieser Eigentümlichkeit von Informationsakten ihren tieferen Sinn.

Von einer Warnung wird überdies nicht nur der Täter negativ betroffen, sondern auch etwaige Angehörige, die ihn aufnehmen. Nicht, dass es nicht schon an und für sich eine große Bürde bedeuten würde, einen Sexualstraftäter in der Familie zu haben und ihn auch noch in der eigenen Wohnung aufzunehmen, leiden die Angehörigen unter den feindseligen Reaktionen ihrer Umwelt. 
Schließlich sollte eine vorausschauende Abwägung auch die langfristigen gesamtgesellschaftlichen Nebenfolgen einer Warnung vor Sexualstraftätern in den Blick nehmen. Während nämlich die Fallzahl bei sexuellem Missbrauch von Kindern seit Jahren rückläufig ist, ${ }^{36}$ bewegt sich die öffentliche Wahrnehmung der Gefährdung von Kindern durch Sexualstraftäter manchmal aus dem Bereich des gesunden Problembewusstseins heraus und neigt zu einer Überbewertung der Gefahr. ${ }^{37}$ Womöglich hängt dies damit zusammen, dass jeder einzelne Fall eine große mediale Ausschlachtung erfährt. Zwar darf die individuelle Tragik jedes einzelnen Verbrechens keinesfalls trivialisiert werden. Nur stellt sich die Frage, ob eine bereits eher zu hohe als zu niedrige Gefahrensensibilität durch eine Warnung noch weiter erhöht werden sollte. Wer in übertrieben großer Angst lebt, könnte zu einem unangemessenen Selbstverzicht auf Freiheit bewogen werden. Alles in allem erfordert eine Warnung der Bevölkerung eine komplexe Abwägung, die nur nach reiflicher Überlegung erfolgen sollte und langfristige Planung sowie eine ausführliche Ermittlung des Sachverhalts erfordert. Wenngleich $\$ 28$ des Verwaltungsverfahrensgesetzes nicht anwendbar ist, erscheint eine Anhörung des Betroffenen vor Veröffentlichung einer Warnung zur Ermöglichung einer besseren Abwägung im Lichte des Grundsatzes der Verhältnismäßigkeit angebracht. Denn ist die Information einmal in der Welt, lassen sich ihre Wirkungen kaum mehr kontrollieren. Aus demselben Grund ist auch die Gewährung vorbeugenden Rechtsschutzes angebracht.

\section{Ausblick}

Nur eine mit kühlem Kopf vorgenommene rationale Abwägung kann zu einem verfassungsmäßigen Ergebnis im Umgang mit entlassenen Sexualstraftätern führen. Die Vorkommnisse in und um Heinsberg zeigen, wie sehr sich die Betriebstemperatur der gegenwärtigen Debatte von diesem Idealzustand entfernt hat. Daran trägt keinesfalls die äußerst erfolgreiche - und an sich auch überaus notwendige - Sensibilisierung weiter gesellschaftlicher Kreise für sexualisierte Gewalt gegen Kinder die Verantwortung. Dass das Thema derartige Emotionen zu entfacht, mag tiefere Ursachen haben. Vielleicht hängt es damit zusammen, dass der Einzelne in einer pluralisierten, durchsäkularisierten und von fundamentaler Verunsicherung geprägten Gesellschaft nur noch an wenigen Orten so sicheren Halt findet wie im familiären Nahbereich, was diesen umso wichtiger und verletzlicher erscheinen lässt.

Es stellt sich damit die Frage, ob und wenn ja welche Maßnahmen denn in gesetzgeberischer Hinsicht mit kühlem Kopf erwogen werden könnten. Zu einer kompletten Reform des Rechts der Sicherungsverwahrung wird es in absehbarer Zeit nicht kommen, wenngleich namhafte Experten dies eindringlich eingefordert haben. ${ }^{38}$ Eine weitere Verschärfung der nachträglichen Sicherungsverwahrung jedoch, wie sie von Justizministerinnen unionsgeführter Länder gefordert

36 Polizeiliche Kriminalstatistik, 55. Ausgabe 2007, Tabelle unten auf S. 137, http://bka.de/pks/pks2007/ download/pks-jb_2007_bka.pdf.

37 Einen empirischen Vergleich von kindlichen Verkehrsopfern und Opfern von Kindesmissbrauch in Bezug auf die Entwicklung von Kriminalitätsstatistik einerseits und Wahrnehmung von Kriminalität andererseits sowie eine Kritik der darauf bezogenen gesetzgeberischen Maßnahmen unternehmen M. Brandenstein/H. Kury, Zur öffentlichen Wahrnehmung von Kriminalität, Kriminalistik 59 (2005), S. 639.

38 Vgl. die Stellungnahmen der Sachverständigen Kinzig und Kreuzer vor dem Rechtsausschuss des Bundestages vom 28.5.2008, http://www.bundestag.de/ausschuesse/a06/anhoerungen/Archiv/37_Jugendstrafrecht-Sichverw/05_Wortprotokoll.pdf. 
wird, ${ }^{39}$ hätte die verfassungsrechtlichen Grenzen zu beachten, die die Rechtsprechung des Bundesverfassungsgerichts bereits konturiert hat. ${ }^{40}$ Danach dürfen sich beispielsweise Versäumnisse im Strafverfahren nicht nach dem Ende der Vollstreckung zu Lasten des Täters auswirken, indem sie in der Entscheidung über die Sicherungsverwahrung in Anschlag gebracht werden und damit gewissermaßen die Verteidigungsstrategie des Täters konterkarieren. ${ }^{41}$ Eine Abschaffung der Voraussetzung, dass während des Vollzugs neue, die Gefährlichkeit begründende Tatsachen auftreten, stößt daher auf verfassungsrechtliche Bedenken. Außerdem verlangt Art. 5 Abs. 1 lit. c der EMRK eine hinreichend konkrete Rückfallgefahr. ${ }^{42}$

Die Diskussion um Alternativen zu Sicherungsverwahrung und staatlichen Warnungen setzt in jedem Fall eine Debatte ohne Vorbehalte voraus. Nur dies ist einer aufgeklärten Gesellschaft würdig. Das Mobilisierungspotenzial des Themas stellt für Entscheidungsträger leider einen hohen Anreiz dar, es zur eigenen Profilierung auszunutzen. Zugleich erhöht es die politischen und persönlichen Risiken für diejenigen, die trotz des „Drucks von der Straße“ nach differenzierten Lösungen suchen. Personenbezogene öffentliche Warnungen sind wegen ihrer unkontrollierbaren Wirkungen und ihrer Nichtrückholbarkeit stets denkbar undifferenzierte Instrumente. Von ihnen sollte daher nicht nur im Fall der Haftentlassung von Sexualstraftätern weitestgehend Abstand genommen werden. ${ }^{43}$

\title{
Jochen Bung
}

\section{Abbau der Defizite von Gefangenen oder Hilfe bei der Lösung ihrer Probleme?}

\author{
Eine kleine Komparatistik zu \71 S. 1 StVollzG und Art. 74 BayStVollzG ${ }^{1}$
}

Die Idee der Resozialisierung, jahrzehntelang bestimmendes Leitprinzip des Strafvollzugs, ist unter Druck geraten. Das hat verschiedene Ursachen, zu denen auch der gegenwärtige Trend zu härteren Strafen (punitive turn) und die Konjunktur des Sicherheitsdenkens gehören. Als Folge der Föderalismusreform liegen nunmehr einige neue Gesetze zum Strafvollzug vor (Bayern, Hamburg, Niedersachsen). Auch diese Gesetze enthalten zum Teil Akzentverschiebungen zu Lasten der Resozialisierung. Bislang hat sich die Kritik an der neuen Gesetzgebung vor allem darauf konzentriert, Veränderungen im Verhältnis von Resozialisierung und Sicherung (Schutz der Allgemeinheit) zu kommentieren. Es findet sich aber noch eine ganz andere Spur, die der ursprünglichen Resozialisierungskonzeption zuwiderläuft. Gemeint sind neue „Hilfs-“ und Behandlungskonzep-

39 Presseerklärung der Ministerinnen Müller-Piepenkötter (NRW) und Merk (Bayern) vom 14.5.2009, http://www.justiz.nrw.de/Presse/PresseJM/14_05_09/index.php.

40 U.a. BVerfGE 109, 133; 109, 190; Beschluss vom 23.8.2006 - 2 BvR 226/06 -.

41 Beschluss vom 23.8.2006 - 2 BvR 226/06 - Rn. 20.

42 O. Dörr, in: Grote/Marauhn (Hrsg.), EMRK. Konkordanz-Kommentar, Kap. 13 Rn. 182.

43 Das fatale Potenzial personenbezogener polizeilicher Informationen konnte jüngst am Beispiel einer HIV-positiven Prominenten besichtigt werden, über deren Verhaftung wegen des Verdachts, einen Liebhaber nicht über ihre Infektion informiert zu haben, die Staatsanwaltschaft ungeniert öffentlich berichtete, vgl. „Der Staatsanwalt in meinem Bett“, FAZ vom 19.4.2009.

1 Vortrag am Fachbereich Rechtswissenschaft der Universität Bremen vom 7. Januar 2009. 